\title{
Sexual dysfunction in patients with schizophrenia treated with conventional antipsychotics or risperidone
}

\author{
Hong Liu-Seifert ${ }^{\prime}$ \\ Bruce J Kinon' \\ Christopher J Tennant ${ }^{2}$ \\ Jennifer Sniadecki' \\ Jan Volavka ${ }^{3}$
}

'Lilly Research Laboratories, Eli Lilly and Company, Lilly Corporate Center, Indianapolis, IN, USA; ${ }^{2} \mathrm{CJT}$ Biomedical Consulting, South Lake Tahoe, CA, USA; ${ }^{3} \mathrm{New}$ York University, New York, NY, USA

Correspondence: Hong Liu-Seifert Lilly Research Laboratories, Eli Lilly and Company, Lilly Corporate Center, Drop Code 6152, Indianapolis, IN 46285, USA

$\mathrm{Tel}+\mathrm{I} 3174330662$

$\mathrm{Fax}+\mathrm{I} 3172766026$

Email liu-seifert_hong@lilly.com
Objective: To better understand sexual dysfunction in patients with schizophrenia and its associations with prolactin and reproductive hormones.

Methods: This was a secondary analysis of an open-label, one-day study $(\mathrm{N}=402)$. The primary objective of the study was to assess the prevalence of hyperprolactinemia in patients with schizophrenia who had been treated with conventional antipsychotics or risperidone. Other atypical antipsychotics available at the time of the study were not included due to a more favorable prolactin profile.

Results: The majority of patients (59\% of females and $60 \%$ of males) reported impairment of sexual function. In postmenopausal females, risk of impaired sexual interest was increased by $31 \%$ for every $10 \mathrm{ng} / \mathrm{ml}$ increase in prolactin $(\mathrm{p}=0.035)$. In males, lower testosterone was associated with higher prolactin $(\mathrm{p}<0.001)$ and with orgasmic $(\mathrm{p}=0.004)$ and ejaculatory dysfunction $(\mathrm{p}=0.028)$.

Conclusion: These findings suggest that hyperprolactinemia may be associated with sexual dysfunction. They also provide more information on the relationships between prolactin, reproductive hormones, and sexual dysfunction. Sexual dysfunction is an understudied yet important consideration in the treatment of schizophrenia. More attention is warranted in this area as it may provide opportunities for improved quality of life and adherence to treatment for patients.

Keywords: sexual dysfunction, schizophrenia, hyperprolactinemia, antipsychotics, risperidone

\section{Introduction}

Sexual dysfunction is commonly experienced by patients with schizophrenia receiving antipsychotic drug treatment and has been reported to occur more frequently with conventional antipsychotics. ${ }^{1-5}$ Treatment-emergent sexual dysfunction in this patient population includes impairment of libido, orgasmic function, and erectile and ejaculatory function. ${ }^{1,6,7}$ While this syndrome has not been extensively researched in large, controlled studies, treatment-emergent sexual dysfunction is known to result in physical and psychological distress and can negatively influence treatment compliance. ${ }^{6-9}$

The relatively high prevalence of sexual dysfunction in patients treated with antipsychotics has been associated with elevations in prolactin, an effect attributed to the acute and chronic dopamine-blocking effect of this drug class. ${ }^{12-15}$ The mechanism by which prolactin elevation influences sexual dysfunction is unclear. Published reports have suggested that elevations in prolactin adversely affect sexual function indirectly via downregulation of estradiol ${ }^{16}$ or testosterone, ${ }^{17,18}$ although others have reported correlations between hyperprolactinemia and sexual dysfunction independent of those hormone levels. ${ }^{19-22}$ 
The development of acute movement disorders such as tardive dyskinesia and extrapyramidal symptoms has also been reported during treatment with antipsychotic medications. ${ }^{23,24}$ These abnormalities have been reported to occur with a higher prevalence during treatment with conventional antipsychotics than with atypical antipsychotics, and their adverse impact on quality of life and treatment compliance has been documented. ${ }^{24-31}$

In addition to treatment-related factors that may be associated with sexual dysfunction, behavioral factors such as use of caffeine, alcohol, and tobacco also are worthy of investigation. Cigarette smoking is known to occur with greater prevalence among patients with schizophrenia than among the adult population overall, and published reports indicate that this population consumes more caffeine daily $y^{32,33}$ and has a higher prevalence of alcohol dependency. ${ }^{34}$

The objective of this research is to better understand the relationships between sexual dysfunction, prolactin, and reproductive hormones. Potential associations between sexual functioning and acute movement disorders as well as behavioral factors including caffeine, alcohol, and tobacco were also explored. These variables are of interest because all may be attributable to some degree to a dopamine $\mathrm{D}_{2}$ receptor blockade. High levels of $\mathrm{D}_{2}$ occupancy have been associated with hyperprolactinemia, extrapyramidal symptoms, as well as worsening of mood and depressive symptoms. ${ }^{35-44}$

\section{Methods}

This was a secondary analysis of a one-day, multicenter, observational study. The primary objective of the study as well as a brief description of the study is provided below. Additional details can be found in the primary report of the study. ${ }^{16}$

\section{Subjects}

This study included patients meeting American Psychiatric Association (APA) criteria for schizophrenia, schizoaffective disorder, or schizophreniform disorder (Diagnostic and Statistical Manual of Mental Disorders, Fourth Edition [DSM-IV; APA, 1994]). Patients must be between 18 and 70 years of age and have received treatment with a conventional antipsychotic drug or risperidone for at least three months prior to entering the study. Medications were prescribed by the patients' psychiatrists using clinical judgment. Conventional antipsychotics and risperidone were included in the study due to their associations with elevated prolactin. Patients taking antipsychotic drugs with a more favorable prolactin profile (clozapine, olanzapine, and quetiapine) or concomitant prolactin-elevating medications were not included. At the time the study protocol was designed and approved (1998), there were no other atypical antipsychotics available. Other key exclusion criteria for the study included pregnancy, nursing, sex hormone therapy, DSM-IV substance abuse or dependency, and thyroid or pituitary gland diseases. Patients who met the above selection criteria during the screening were scheduled to enter the one-day clinical visit after their informed consent documents have been received. Each study site's Institutional Review Board approved the informed consent documents as well as the study protocol, which was developed in accordance with the ethical standards of good clinical practice and the Declaration of Helsinki.

\section{Study design}

This study was conducted at 27 centers in the United States from September 1998 through May 2000. Patients were requested to have abstained from sexual activity within 12 hours prior to the visit. Patients arrived at the study center in the morning, and blood was collected for measurement of serum prolactin, estradiol in females, and total and free testosterone in males. Patients were given a small bland meal, and a second blood sample was collected three to four hours later from which a second prolactin measurement was obtained. Each patient's prolactin level was considered as the mean value from the two blood samples.

\section{Assay methodology}

Whole blood samples were centrifuged to separate serum. Serum was shipped at ambient temperature on the day of collection and assayed by Covance Central Laboratory (Indianapolis, IN). Prolactin was assayed by microparticle enzyme immunoassay (sensitivity $0.60 \mathrm{ng} / \mathrm{mL}$, intra-assay coefficients of variation [c.v.] 3.2\%-3.6\%, inter-assay c.v. 4.5\%-5.2\%). Testosterone was measured using Coat-a-Count radioimmunoassay procedure (assay sensitivity $8.0 \mathrm{ng} / \mathrm{dL}$, intra-assay c.v. $11.2 \%$, inter-assay c.v. $11.0 \%$ ). Estradiol was measured using a double-antibody radioassay procedure (sensitivity $1.4 \mathrm{pg} / \mathrm{mL}$, intra-assay c.v. $6.5 \%$, inter-assay c.v. $7.6 \%)$.

\section{Assessment of sexual dysfunction}

The Global Impression of Sexual Function (GISF) is a self-rated scale by patient that measures a subject's feelings toward his or her sexual function. The GISF is designed to assess sexual interest/desire, arousal (as characterized by penile erection or vaginal lubrication), orgasm, and overall sexual function. Each item was rated from 1 ("normal") to 5 ("severely impaired"). 
The Changes in Sexual Functioning Questionnaire (CSFQ) is a clinician-rated, structured interview/questionnaire designed to measure dysfunction and changes in sexual function during treatment with medication. ${ }^{45}$ The CSFQ, which consists of 36 questions for men and 35 questions for women, assesses components of sexuality pertaining to pleasure, desire/frequency, desire/interest, arousal, and orgasm.

\section{Assessment of extrapyramidal symptoms}

The Simpson-Angus Scale ${ }^{46}$ is an assessment tool used to measure treatment-emergent parkinsonism. The modified version of the scale used in this study eliminated items relating to head drop and leg pendulousness; combined items pertaining to shoulder shaking, elbow rigidity, and fixation of position; and added items on balance, akinesia, akathisia, acute dystonic reaction, and cooperativeness.

The Barnes Akathisia Scale ${ }^{47}$ was used to assess observable movements of treatment-emergent akathisia, subjective awareness and distress associated with akathisia, and provide a global clinical assessment of severity.

The Abnormal Involuntary Movement Scale (AIMS) ${ }^{48}$ was used to record the occurrence of dyskinetic movements in specific body regions and assess the severity, level of incapacitation, and subject's awareness of the movements.

\section{Assessment of caffeine/alcohol/tobacco use}

Patients' past and current use of caffeine, alcohol, and tobacco was obtained from medical histories and subjects' self-reports. Specifically, the current use was assessed through questions with regard to the weekly consumption of alcohol including beer, spirits, and wine; total daily use of tobacco; number of years smoked; and average daily consumption of caffeine.

\section{Statistical methods}

Patient characteristics were tabulated by gender. Fisher's exact test was used to compare male and female patients' social and reproductive histories and responses to sexual functioning questionnaires. Fisher's exact test was also used to compare hyperprolactemia status among females. Hyperprolactinemia was defined as a prolactin level above the upper limit of normal $(0.8 \mathrm{nmol} / \mathrm{L}$ [18.77 $\mathrm{ng} / \mathrm{mL}]$ for males and $1.05 \mathrm{nmol} / \mathrm{L}$ [24.20 ng/mL] for females). Different aspects of sexual dysfunction were defined using a criterion of a score $\geq 3$ on each of the four GISF items and analyzed separately for males and females.
The effect of prolactin level on different aspects of sexual dysfunction was modeled using a logistic regression model with prolactin level as the independent variable. The effects of estradiol (for females) and free and total testosterone (for males) on sexual functioning based on both GISF and CSFQ were also analyzed with a logistic regression model after adjusting for age. In addition, logistic regression analyses were conducted to assess the dose response relationship associated with sexual dysfunction. The association of prolactin level and sex hormones were tested using a Pearson correlation test.

Linear regression was used to examine the relationship between sexual dysfunction and acute movement disorders after adjusting for age. Each item of the Simpson-Angus Scale, Barnes Akathisia Scale, and AIMS were used as individual predictors. The association of prolactin level and acute movement disorders was tested using Spearman's rank correlation test. Multiple linear regression was used to test the relationship between sexual dysfunction and behavioral habits. The number of caffeinated drinks (daily), cigarettes (cigars, pipes: daily), or alcoholic drinks (weekly) were used as individual predictors after adjusting for age.

As the analyses in this research were secondary and exploratory in nature, no corrections for multiple comparisons were made.

\section{Results}

\section{Demographics and disease characteristics}

Patient demographics and disease characteristics are summarized by gender in Table 1. Use of specific antipsychotic drugs and their doses are also presented in the table. The majority of subjects were Caucasian. Mean age was $40.8( \pm 10.0)$ for males and $44.5( \pm 11.4)$ for females. Approximately one-third of subjects were receiving risperidone, and two-thirds were receiving conventional antipsychotics. Of the female subjects, $61 \%$ were premenopausal, $35 \%$ were postmenopausal, and $4 \%$ were perimenopausal or of unknown status. Hyperprolactinemia was present in $42 \%$ of males and $59 \%$ of females, including $66 \%$ of premenopausal females and $45 \%$ of postmenopausal females.

Patients' social and reproductive history is summarized by gender. Relatively few subjects ( $8 \%$ of males and $19 \%$ of females) described themselves as married or cohabitating. Education history and current employment were similar for male and female patients. Approximately one-quarter of patients were high school graduates but fewer than $10 \%$ were 
Table I Patient and disease characteristics

\begin{tabular}{|c|c|c|}
\hline & Males $(n=255)$ & Females $(n=147)$ \\
\hline Age $($ mean $\pm S D)$ & $40.8 \pm 10.0$ & $44.5 \pm 11.4$ \\
\hline \multicolumn{3}{|l|}{ Race (n/\%) } \\
\hline Caucasian & $124(48.6 \%)$ & $62(42.2 \%)$ \\
\hline African descent & $96(37.6 \%)$ & $68(46.2 \%)$ \\
\hline Asian descent & $7(2.7 \%)$ & $6(4.1 \%)$ \\
\hline Other & $28(11.0 \%)$ & II (7.5\%) \\
\hline \multicolumn{3}{|l|}{ Menopausal Status (n/\%) } \\
\hline Premenopausal & & $90(61.2 \%)$ \\
\hline Postmenopausal & & $51(34.7 \%)$ \\
\hline Perimenopausal/unknown & & $6(4.0 \%)$ \\
\hline \multicolumn{3}{|l|}{ Serum prolactin ${ }^{a}$} \\
\hline Below upper limit of normal ${ }^{b}$ & $147(57.6 \%)$ & $60(40.8 \%)$ \\
\hline Elevated & $108(42.4 \%)$ & 87 (59.2\%) \\
\hline \multicolumn{3}{|l|}{ Diagnosis } \\
\hline Schizophrenia & $85.5 \%$ & $76.2 \%$ \\
\hline Schizoaffective & $14.5 \%$ & $23.8 \%$ \\
\hline Length of illness (yrs $\pm S D$ ) & $17.2 \pm 9.7$ & $18.7 \pm 11.3$ \\
\hline \multicolumn{3}{|l|}{ Drug $\mathrm{n} /$ mean dose (mg/day) } \\
\hline Risperidone & $84 / 5.2$ & $42 / 4.7$ \\
\hline \multicolumn{3}{|l|}{ Conventional - Oral } \\
\hline Chlorpromazine & $7 / 157.14$ & $3 / 266.67$ \\
\hline Fluphenazine & $1 \mathrm{I} / 2 \mathrm{I} .32$ & $12 / 13.06$ \\
\hline Haloperidol & $28 / 14.32$ & $16 / 8.69$ \\
\hline Loxapine & $4 / 86.25$ & $3 / 41.67$ \\
\hline Mesoridazine & $3 / 166.67$ & $2 / 27.50$ \\
\hline Molindone & $\mathrm{I} / \mathrm{I} 25.00$ & 0 \\
\hline Perphenazine & $5 / 8.40$ & $9 / 14.44$ \\
\hline Pimozide & $1 / 4.00$ & 0 \\
\hline Thioridazine hydrochloride & $14 / 294.64$ & II/295.45 \\
\hline Tiotixene & $10 / 21.50$ & $5 / 8.00$ \\
\hline Trifluoperazine & $3 / 23.33$ & $5 / 14.40$ \\
\hline \multicolumn{3}{|l|}{ Conventional - depot (mg/month) } \\
\hline Fluphenazine decanoate & $43 / 74.4$ & $18 / 60.94$ \\
\hline Haloperidol decanoate & $4 I / / 38.5$ & $20 / 133.45$ \\
\hline
\end{tabular}

Notes: ${ }^{a}$ Only patients whose serum prolactin and reproductive hormone values were available are included; ${ }^{\mathrm{T}}$ The upper limit of normal (ULN) for serum prolactin was defined as $18.77 \mathrm{ng} / \mathrm{mL}$ for males and $24.20 \mathrm{ng} / \mathrm{mL}$ for females.

college graduates, and more than $80 \%$ were unemployed at the time of the study. Fewer male patients than female patients reported biologic offspring (33\% compared with $57 \%, \mathrm{p}<0.001)$.

\section{Prevalence of sexual dysfunction}

Majority of patients (59\% of females and $60 \%$ of males) reported impairment of overall sexual function. The prevalence rate of overall sexual dysfunction did not differ based on type of drug treatment (risperidone vs conventional antipsychotics), regardless of whether the population was analyzed overall or by gender.

\section{Association between age and sexual dysfunction}

The relationship between age and sexual dysfunction was significant for male patients (odds ratio $[\mathrm{OR}]=1.04 ; 95 \%$ confidence interval [CI] [1.01-1.07], $\mathrm{p}=0.003)$, indicating that the odds of sexual dysfunction was increased by $40 \%$ with each 10-year age increase in males. The correlation between 
age and sexual dysfunction did not reach statistical significance for females ( $\mathrm{OR}=1.02$; CI [0.96, 1.05], $\mathrm{p}=0.29)$.

\section{Association between dose and sexual dysfunction}

A significant dose response was observed in males on risperidone treatment (Figure 1), among whom the odds of impaired orgasm was increased by $26 \%(\mathrm{OR}=1.26 \% ; 95 \% \mathrm{CI}$ [1.03-1.54]; $\mathrm{p}=0.02$ ) for every $1 \mathrm{mg}$ increase in risperidone. Otherwise, significant relationships between drug dose and any aspect of sexual functioning (sexual interest/desire, vaginal lubrication/erection, or overall) were not observed in any group of patients (males, premenopausal females, or postmenopausal females) regardless of type of drug treatment.

\section{Association between elevated prolactin and sexual dysfunction}

An increase in prolactin level in female patients was associated with greater odds of impaired sexual interest $(\mathrm{OR}=1.009 ; 95 \%$ CI $[1.000-1.019] ; \mathrm{p}=0.052)$. This effect was driven primarily by postmenopausal females, among whom the odds of impaired sexual interest are increased by $31 \%(\mathrm{OR}=1.031 ; 95 \%$ CI $[1.002-1.060] ; \mathrm{p}=0.035)$ for every $10 \mathrm{ng} / \mathrm{mL}$ increase in prolactin.

Elevated prolactin was not associated with sexual dysfunction among males in the overall population or among males treated with conventional antipsychotics. However, an association between elevated prolactin and impairment of orgasm was observed among males treated with risperidone $(\mathrm{OR}=1.031 ; 95 \% \mathrm{CI}[1.002-1.062] ; \mathrm{p}=0.039)$.

\section{Role of estradiol and testosterone in sexual dysfunction}

When adjusted for age, estradiol level did not have a significant effect on any aspect of sexual functioning in female patients. Among male patients, both total and free testosterone were negatively correlated with prolactin level (correlation coefficient $=-0.22$ and $-0.28, \mathrm{p}=0.0003$ and $\mathrm{p}=0.0001$, respectively). When adjusted for age, lower concentration of total testosterone was associated with an inability to ejaculate when desired $(p=0.028)$, and lower concentration of free testosterone was associated with painful orgasm ( $p=0.004$; Table 2 ) based on CSFQ measures.

\section{Association between acute movement disorders and sexual dysfunction}

In male patients after adjusting for age, impairment of sexual function was associated with involuntary movement of the lower body, impaired balance, excessive salivation, akinesia, and akathisia. Age was associated with worse outcomes on multiple measures of the acute movement scales. Due to the size, data are not presented here and will be available upon request.

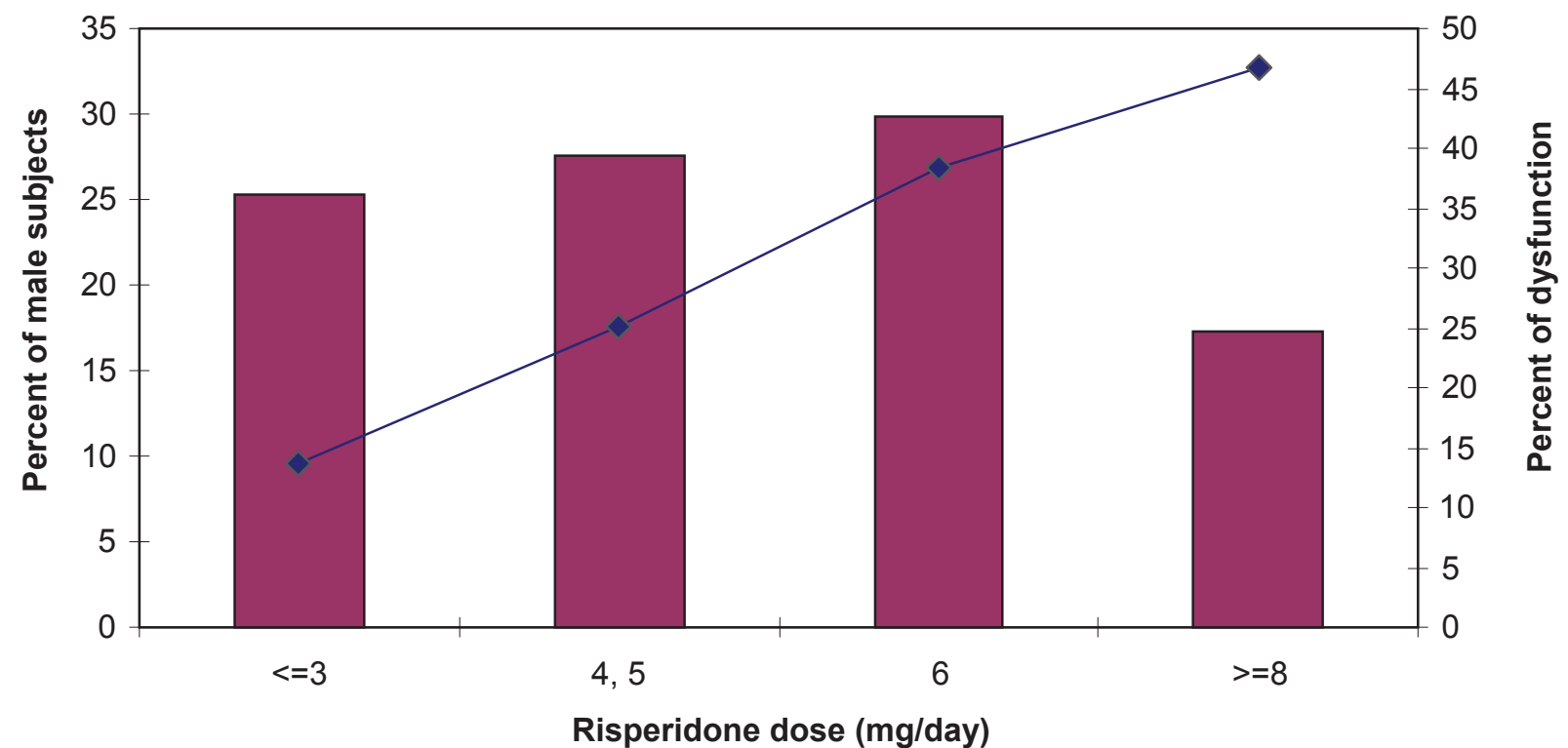

Risperidone dose (mg/day)

Male subjects $\quad \longrightarrow$ Impaired orgasm

Figure I Prevalence of impaired orgasm by risperidone dose in male subjects $(n=87)$. Impairment of orgasm was defined as a score $\geq 3$ on the orgasm component of the Global Impressions of Sexual Function questionnaire. 
Table 2 Age-adjusted association between serum testosterone and sexual dysfunction in male subjects

\begin{tabular}{|c|c|c|c|c|c|c|c|}
\hline \multirow[t]{2}{*}{ CSFQ Item } & \multirow{2}{*}{$\begin{array}{l}\text { Prevalence } \\
\text { of dysfunction }\end{array}$} & \multicolumn{3}{|c|}{ Total testosterone } & \multicolumn{3}{|c|}{ Free testosterone } \\
\hline & & p value ${ }^{a}$ & Odds ratio & $95 \% \mathrm{Cl}$ & p value ${ }^{a}$ & Odds ratio & $95 \% \mathrm{Cl}$ \\
\hline Ejaculate when wanted & $20.2 \%$ & 0.028 & 0.998 & $0.996,1.000$ & 0.102 & 0.954 & $0.902,1.009$ \\
\hline Painful orgasm & $6.3 \%$ & 0.455 & 1.001 & $0.998,1.004$ & 0.004 & I.I 175 & $1.052,1.312$ \\
\hline
\end{tabular}

Abbreviations: $\mathrm{Cl}$, confidence interval; CSFQ, Changes in Sexual Functioning Questionnaire.

Notes: "P values were obtained by logistic regression. As measured by CSFQ, sexual dysfunction was defined as a score of I or 2 for "ejaculate when wanted", and a score of 3 or greater for "painful orgasm."

In female patients after adjusting for age, impairment of sexual function was associated with involuntary movement of facial expression muscles and impaired balance. Age was associated with a worse outcome on a single component of the modified Simpson-Angus scale. Due to the size, data are not presented here and will be available upon request.

An examination of the possible association between prolactin level and acute movement disorders revealed the associations between prolactin and the three movement scales were not statistically significant for both males and females.

\section{Association between caffeine/alcohol/ tobacco use and sexual dysfunction}

In male patients after adjusting for age, daily smoking (daily number of cigarettes and/or total daily smoking) and years smoked were both associated with better scores in erections, orgasm, and overall sexual function, and greater number of years smoked was associated with a higher degree of sexual interest/desire. Sexual dysfunction was not associated with alcohol or caffeine use in either males or females.

\section{Discussion}

Data presented in this paper revealed a sexual dysfunction prevalence rate of approximately $60 \%$, regardless of gender, which was consistent with published reports. ${ }^{1,7,9}$ Elevated prolactin levels were found to be associated with sexual dysfunction.

Some reports have suggested that sexual dysfunction ultimately results from prolactin-mediated down-regulation of serum testosterone or estradiol. ${ }^{16-18}$ Data in this research indicate that prolactin elevations in males were correlated with lower levels of total and free serum testosterone. Those reductions, in turn, were associated with dysfunction relating to orgasm and ejaculation. This suggests that the association between hyperprolactinemia and sexual dysfunction in males may be mediated by reductions in testosterone.

Prolactin elevations also were associated with impaired sexual interest in postmenopausal women. The associations between prolactin and estradiol, and between estradiol and sexual dysfunction, were not significant.

Few reports have addressed the possible association between acute movement disorders associated with antipsychotic treatment and sexual dysfunction in patients with schizophrenia. Our data indicate an association between various components of sexual dysfunction and movement disorders relating to tardive dyskinesia, parkinsonism, and akathisia. The underlying mechanism of this potential association is not well understood. As prolactin was not associated with movement disorders, it is not likely a mediator for the relationship between movement and sexual functions. Further studies are needed to confirm the association seen between movement disorders and sexual dysfunction and to explore the potential underlying mechanism.

One unexpected outcome in this research was that tobacco smoking was significantly correlated with better sexual functioning in males. This finding was somewhat surprising, given that smoking has been associated with male erectile dysfunction. ${ }^{49}$ However, tobacco smoking has been linked to enhancing the metabolism and excretion of antipsychotic agents. ${ }^{50,51}$ Thus, it may be possible that the association between tobacco smoking and better sexual functioning in males was attributable to faster clearance of the antipsychotic agents in those patients. Future studies are needed to further explore this potential theory.

An important limitation that needs discussion is that the study did not include other atypical antipsychotic drugs than risperidone. As the primary objective of the study was to evaluate prevalence of hyperprolactinemia, only conventional antipsychotics and risperidone were included in the study due to their associations with elevated prolactin levels. Atypical antipsychotic drugs with a more favorable prolactin profile (clozapine, olanzapine, and quetiapine) were not included. There were no other atypical antipsychotics available at the time the study protocol was designed and approved (1998). Prospective and controlled future studies to facilitate the comparison 
of risperidone and conventionals to antipsychotics with a more favorable prolactin profile are needed and will help to better ascertain the effect of prolactin on sexual functioning.

A second limitation of the present research is that CSFQ has not been specifically validated in schizophrenia patients treated with antipsychotics. The Psychotropic-Related Sexual Dysfunction (PRSexDQ-SALSEX) is a brief and relatively nonintrusive questionnaire and has been recently validated in a sample of patients with schizophrenia or other psychotic disorders who were experiencing anti-psychotic-induced sexual dysfunction and were switched to olanzapine. ${ }^{52}$

Another limitation is that the study was designed to be a one-day clinic visit, thus, only allowing a cross-sectional examination of prolactin level and its association with sexual dysfunction. Prospective studies that follow patients over a period of time will be able to provide further information on the time course of the development of sexual dysfunction and the effect of prolactin on sexual dysfunction over time.

Overall, these data suggest that elevated prolactin was associated with sexual dysfunction. They also provide more information on the relationships between prolactin, reproductive hormones, and sexual functioning in patients with schizophrenia. Sexual dysfunction is an understudied yet important consideration in the treatment of schizophrenia. More attention is warranted in this area as it may provide opportunities for improved quality of life and adherence to treatment for patients. ${ }^{6-9}$

\section{Disclosure}

This work was sponsored by Eli Lilly and Company.

\section{References}

1. Ucok A, Incesu C, Aker T, et al. Sexual dysfunction in patients with schizophrenia on antipsychotic medication. Eur Psychiatry. 2007;22:328-333.

2. Knegtering H, van der Moolen AE, Castelein S, et al. What are the effects of antipsychotics on sexual dysfunctions and endocrine functioning? Psychoneuroendocrinology. 2003;28:109-123.

3. Knegtering $\mathrm{H}$, Boks M, Blijd C, et al. A randomized open-label comparison of the impact of olanzapine versus risperidone on sexual functioning. J Sex Marital Ther. 2006;32:315-326.

4. Kim KS, Pae CU, Chae JH, et al. Effects of olanzapine on prolactin levels of female patients with schizophrenia treated with risperidone. J Clin Psychiatry. 2002;63:408-413.

5. Dossenbach M, Hodge A, Anders M, et al. Prevalence of sexual dysfunction in patients with schizophrenia: international variation and underestimation. Int J Neuropsychopharmacol. 2005;8:195-201.

6. Costa AM, Lima MS, Mari Jde J. A systematic review on clinical management of antipsychotic-induced sexual dysfunction in schizophrenia. Sao Paulo Med J. 2006;124:291-297.

7. Olfson M, Uttaro T, Carson WH, et al. Male sexual dysfunction and quality of life in schizophrenia. J Clin Psychiatry. 2005;66:331-338.

8. Cutler AJ. Sexual dysfunction and antipsychotic treatment. Psychoneuroendocrinology. 2003;28(Suppl 1):69-82.
9. Rosenberg KP, Bleiberg KL, Koscis J, et al. A survey of sexual side effects among severely mentally ill patients taking psychotropic medications: impact on compliance. J Sex Marital Ther. 2003; 29:289-296

10. Haddad PM, Wieck A. Antipsychotic-induced hyperprolactinaemia: mechanisms, clinical features and management. Drugs. 2004;64:2291-2314.

11. Ghadirian AM, Chouinard G, Annable L. Sexual dysfunction and plasma prolactin levels in neuroleptic-treated schizophrenic outpatients. JNerv Ment Dis. 1982;170:463-467.

12. Breier AF, Malhotra AK, Su TP, et al. Clozapine and risperidone in chronic schizophrenia: effects on symptoms, parkinsonian side effects, and neuroendocrine response. Am J Psychiatry. 1999;156: 294-298.

13. Crawford AM, Beasley CM Jr, Tollefson GD. The acute and long-term effect of olanzapine compared with placebo and haloperidol on serum prolactin concentrations. Schizophr Res. 1997;26:41-54.

14. Rubin RT. Prolactin and schizophrenia. In: Meltzer HY, editor. Psychopharmacology: The Third Generation. New York: Raven Press; 1987; p. 803-808.

15. Halbreich U, Kinon BJ, Gilmore JA, et al. Elevated prolactin levels in patients with schizophrenia: mechanisms and related adverse effects. Psychoneuroendocrinology. 2003;28(Suppl 1):53-67.

16. Kinon BJ, Gilmore JA, Liu H, et al. Prevalence of hyperprolactinemia in schizophrenic patients treated with conventional antipsychotic medications or risperidone. Psychoneuroendocrinology. 2003;28:55-68.

17. Schwartz MF, Bauman JE, Masters WH. Hyperprolactinemia and sexual disorders in men. Biol Psychiatry. 1982;17:861-876.

18. Lundberg PO, Hulter B. Sexual dysfunction in patients with hypothalamopituitary disorders. Exp Clin Endocrinol. 1991;98:81-88.

19. Buvat J. Hyperprolactinemia and sexual function in men: a short review. Int J Impot Res. 2003;15:373-377.

20. Buvat J, Lemaire A, Buvat-Herbaut M, et al. Hyperprolactinemia and sexual function in men. Horm Res. 1985;22:196-203.

21. Kadioglu P, Yalin AS, Tiryakioglu O, et al. Sexual dysfunction in women with hyperprolactinemia: a pilot study report. J Urol. 2005;174:1921-1925.

22. Turna B, Apaydin E, Semerci B, et al. Women with low libido: correlation of decreased androgen levels with female sexual function index. Int J Impot Res. 2005; 17:148-153.

23. Casey DE. Tardive dyskinesia and atypical antipsychotic drugs. Schizophr Res. 1999;35(Suppl):S61-S66.

24. Caroff SN, Mann SC, Campbell EC, et al. Movement disorders associated with atypical antipsychotic drugs. J Clin Psychiatry. 2002;63(Suppl 4):12-19.

25. Tenback DE, van Harten PN, Slooff CJ, et al. Effects of antipsychotic treatment on tardive dyskinesia: a 6-month evaluation of patients from the European Schizophrenia Outpatient Health Outcomes (SOHO) Study. J Clin Psychiatry. 2005;66:1130-1133.

26. Weiden PJ. EPS profiles: the atypical antipsychotics are not all the same. J Psychiatr Pract. 2007;13:13-24.

27. Collaborative Working Group on Clinical Trial Evaluations. Adverse effects of the atypical antipsychotics. J Clin Psychiatry. 1998;59(Suppl 12):17-22.

28. Altamura AC, Mauri MC, De Novellis F, et al. Residual neurolepticinduced parkinsonian symptoms in schizophrenia. A naturalistic study with orphenadrine. Pharmacopsychiatry. 1989;22:246-249.

29. Glazer WM, Morgenstern H, Doucette JT. Predicting the long-term risk of tardive dyskinesia in outpatients maintained on neuroleptic medications. J Clin Psychiatry. 1993;54:133-139.

30. Morgenstern H, Glazer WM. Identifying risk factors for tardive dyskinesia among long-term outpatients maintained with neuroleptic medications. Results of the Yale Tardive Dyskinesia Study. Arch Gen Psychiatry. 1993;50:723-733.

31. Krebs M, Leopold K, Hinzpeter A, et al. Current schizophrenia drugs: efficacy and side effects. Expert Opin Pharmacother. 2006; 7:1005-1016. 
32. Strassnig M, Brar JS, Ganguli R. Increased caffeine and nicotine consumption in community-dwelling patients with schizophrenia. Schizophr Res. 2006;86:269-275.

33. Henderson DC, Borba CP, Daley TB, et al. Dietary intake profile of patients with schizophrenia. Ann Clin Psychiatry. 2006;18:99-105.

34. Regier DA, Farmer ME, Rae DS, et al. Comorbidity of mental disorders with alcohol and other drug abuse. Results from the Epidemiologic Catchment Area (ECA) Study. JAMA. 1990;264:2511-2518.

35. Kapur S, Zipursky R, Jones C, et al. Relationship between dopamine $\mathrm{D}(2)$ occupancy, clinical response, and side effects: a double-blind PET study of first-episode schizophrenia. Am J Psychiatry. 2000;157:514-520.

36. Kapur S, Remington $G$, Jones $C$, et al. High levels of dopamine $D_{2}$ receptor occupancy with low-dose haloperidol treatment: a PET study. Am J Psychiatry. 1996;153:948-950.

37. Nordström AL, Farde L, Wiesel FA, et al. Central $\mathrm{D}_{2}$-dopamine receptor occupancy in relation to antipsychotic drug effects: a double-blind PET study of schizophrenic patients. Biol Psychiatry. 1993;33:227-235.

38. Kapur S, Zipursky RB, Remington G. Clinical and theoretical implications of 5-HT2 and $\mathrm{D}_{2}$ receptor occupancy of clozapine, risperidone, and olanzapine in schizophrenia. Am J Psychiatry. 1999;156:286-293.

39. Schlegel S, Schlösser R, Hiemke C, et al. Prolactin plasma levels and $\mathrm{D}_{2}$-dopamine receptor occupancy measured with IBZM-SPECT. Psychopharmacology (Berl). 1996;124:285-287.

40. Farde L, Nordström AL, Wiesel FA, et al. Positron emission tomographic analysis of central $\mathrm{D}_{1}$ and $\mathrm{D}_{2}$ dopamine receptor occupancy in patients treated with classical neuroleptics and clozapine. Relation to extrapyramidal side effects. Arch Gen Psychiatry. 1992;49:538-544.

41. Mizrahi R, Rusjan P, Agid O, et al. Adverse subjective experience with antipsychotics and its relationship to striatal and extrastriatal D2 receptors: a PET study in schizophrenia. Am J Psychiatry. 2007;164:630-637.
42. Saeedi H, Remington G, Christensen BK. Impact of haloperidol, a dopamine D2 antagonist, on cognition and mood. Schizophr Res. 2006;85:222-231.

43. de Haan L, Lavalaye J, Linszen D, et al. Subjective experience and striatal dopamine $\mathrm{D}(2)$ receptor occupancy in patients with schizophrenia stabilized by olanzapine or risperidone. Am J Psychiatry. 2000;157:1019-1020.

44. Bressan RA, Costa DC, Jones HM, et al. Typical antipsychotic drugs $\mathrm{D}(2)$ receptor occupancy and depressive symptoms in schizophrenia. Schizophr Res. 2002;56:31-36.

45. Clayton AH, McGarvey EL, Clavet GJ. The Changes in Sexual Functioning Questionnaire (CSFQ): development, reliability, and validity. Psychopharmacol Bull. 1997;33:731-745.

46. Simpson GM, Angus JW. A rating scale for extrapyramidal side effects. Acta Psychiatr Scand Suppl. 1970;212:11-19.

47. Barnes TR. A rating scale for drug-induced akathisia. Br J Psychiatry. 1989;154:672-676.

48. Munetz MR, Benjamin S. How to examine patients using the Abnormal Involuntary Movement Scale. Hosp Community Psychiatry. 1988,39:1172-1177.

49. Dorey G. Is smoking a cause of erectile dysfunction? A literature review. Br J Nurs. 2001;10:455-465.

50. Goff DC, Henderson DC, Amico E. Cigarette smoking in schizophrenia: relationship to psychopathology and medication side effects. $\mathrm{Am}$ J Psychiatry. 1992;149:1189-1194.

51. van der Weide J, Steijns LS, van Weelden MJ. The effect of smoking and cytochrome P450 CYP1A2 genetic polymorphism on clozapine clearance and dose requirement. Pharmacogenetics. 2003;13:169-172.

52. Montejo AL, Rico-Villademoros F. Psychometric properties of the Psychotropic-Related Sexual Dysfunction Questionnaire (PRSexDQSALSEX) in patients with schizophrenia and other psychotic disorders. J Sex Marital Ther. 2008;34:227-239. 